\title{
A toolkit for rapid gene mapping in the nematode Caenorhabditis briggsae
}

\author{
Daniel C Koboldt ${ }^{1}$, Julia Staisch¹, Bavithra Thillainathan², Karen Haines², Scott E Baird³, Helen M Chamberlin4, \\ Eric S Haag55, Raymond D Miller ${ }^{1}$ and Bhagwati P Gupta*2
}

\begin{abstract}
Background: The nematode C. briggsae serves as a useful model organism for comparative analysis of developmental and behavioral processes. The amenability of C. briggsae to genetic manipulations and the availability of its genome sequence have prompted researchers to study evolutionary changes in gene function and signaling pathways. These studies rely on the availability of forward genetic tools such as mutants and mapping markers.

Results: We have computationally identified more than 30,000 polymorphisms (SNPs and indels) in C. briggsae strains AF16 and HK104. These include 1,363 SNPs that change restriction enzyme recognition sites (snip-SNPs) and 638 indels that range between $7 \mathrm{bp}$ and $2 \mathrm{~kb}$. We established bulk segregant and single animal-based PCR assay conditions and used these to test 107 polymorphisms. A total of 75 polymorphisms, consisting of 14 snip-SNPs and 61 indels, were experimentally confirmed with an overall success rate of $83 \%$. The utility of polymorphisms in genetic studies was demonstrated by successful mapping of 12 mutations, including 5 that were localized to sub-chromosomal regions. Our mapping experiments have also revealed one case of a misassembled contig on chromosome 3.
\end{abstract}

Conclusions: We report a comprehensive set of polymorphisms in C. briggsae wild-type strains and demonstrate their use in mapping mutations. We also show that molecular markers can be useful tools to improve the C. briggsae genome sequence assembly. Our polymorphism resource promises to accelerate genetic and functional studies of $C$. briggsae genes.

\section{Background}

Comparative analysis of developmental and behavioral processes in closely related species is a powerful approach to understand the mechanisms of evolution. It facilitates identification of molecular components that are conserved over millions of years due to their role in specifying common features as well as those that are variable because they confer species-specific features. The model organism Caenorhabditis elegans (a nematode) and its congener, C. briggsae, are particularly suitable for such investigations. Their many experimental advantages include rapid growth, small size, transparency, ease of culture and genetic manipulations, and the availability of fully sequenced genomes [1-3].

C. briggsae is phenotypically almost indistinguishable from C. elegans and has a similar (hermaphroditic) repro-

\footnotetext{
* Correspondence: guptab@mcmaster.ca

2 Department of Biology, McMaster University, Hamilton, ON L8S 4K1, Canada $\wedge$ Deceased

Full list of author information is available at the end of the article
}

ductive mode. The last common ancestor of these two species lived about 30 million years ago [4], and despite the rapid molecular evolution typical of the family Rhabditidae, more than half $(\sim 52 \%)$ of the C. elegans genome aligns with the $C$. briggsae genome assembly [2]. This includes two-thirds of all C. briggsae genes $(13,107$ or $67.8 \%)$ with reciprocal orthologs in C. elegans [5]. Thus C. elegans-C. briggsae comparative genomic and genetic studies promise powerful new tools for the identification of genes and pathways and the study of both conservation and divergence.

Like C. elegans, C. briggsae has six chromosomes that display extensive conservation of synteny, but not exact colinearity relative to C. elegans [6]. While C. briggsae shares many of the experimental advantages of C. elegans, it has the further advantage of increased natural variability for single nucleotide polymorphisms (SNPs) and insertion-deletions (indels) [7,8]. This elevated natural variation potentially enhances its use for genotype-phe- 
notype association studies, and is also very useful for the mapping aspects of forward genetics projects.

Initial work on gene function in C. briggsae employed cross-species transgene rescue of $C$. elegans mutants (e.g. [9-13]) and RNA interference (RNAi; e.g. [13-17]). However, a number of laboratories are now generating true mutations in C. briggsae, using both forward mutagenesis screens [18-20] (R.E. Ellis, personal communication; B.P. Gupta, unpublished results; H. Chamberlin, unpublished results) and PCR-based deletion mutation screens [20]. Positional cloning of C. briggsae mutations without relying upon obvious candidate genes requires a set of mapping tools. Development of such tools is facilitated by a high-quality whole-genome shotgun assembly [2] and the organization of most of the resulting contigs into chromosomes via a SNP-based recombination map [6].

Among the tools needed to facilitate forward genetics in C. briggsae, a set of easily scored DNA polymorphisms is especially important. Experimentally validated polymorphisms can serve as useful markers for mapping mutations that cause visible phenotypes. Additionally, these markers can be integrated with the phenotypebased genetic linkage map (e.g., $d p y$ and $u n c$ mutants [21]) to further enhance their utility. Integration of polymorphisms and phenotype-based maps increases map density and anchors the relative locations of molecular and phenotypic markers. With this goal in mind we have discovered a large set of genome-wide polymorphisms (SNPs and indels) in wild-type strains, using AF16 as a reference strain and four other natural isolate strains: HK104, HK105, VT847, and PB800.

The indels were placed into three classes: small (7-49 bp), medium (50-2,000 bp), and large ( $>2 \mathrm{~kb})$. We have focused on medium and small indels (212 and 7,530, respectively), which offer the greatest utility as genetic markers. In the case of SNPs $(23,829)$, we found that 4,700 modify restriction enzyme sites (termed snip-SNPs) and therefore can be easily detected as restriction fragment length polymorphisms (RFLPs). We established assay conditions for bulk segregant analysis (BSA) and used these to experimentally validate 14 snip-SNPs, 28 medium and 32 small indels. The validated polymorphisms were used to genetically map known mutations causing visible phenotypes thus demonstrating the effectiveness of the polymorphisms in linkage mapping studies. We also developed single animal-based PCR assay to determine map distance. Five mutations were successfully localized to sub-chromosomal regions by 3 or more indels, greatly facilitating the search for each candidate gene. These results demonstrate the utility of our mapping toolkit in genetic linkage and gene identification studies.

\section{Results}

\section{SNP Discovery}

We performed SNP discovery in four C. briggsae strains by aligning paired shotgun sequence reads to the AF16based reference sequence (cb25 assembly, [22]). These sequences were obtained by capillary gel electrophoresis at Washington University Genome Center (see Methods). To build on previous SNP discovery efforts [6], we applied the ssahaSNP algorithm, which detects SNPs and small indels based on SSAHA alignments to a reference sequence (see Methods). Compared to AF16, ssahaSNP detected 23,829 unique SNP loci in HK104 DNA, or one substitution per $163 \mathrm{bp}$ on average (Table 1 and additional file 1). Consistent with $C$. briggsae clade structure [8], SNP density was slightly lower in strains HK105 (1/ $168 \mathrm{bp})$ and PB800 (1/197 bp) and much lower in strain VT847 (1/475 bp). In HK104, the most common substitu-

Table 1: SNPs in various C. briggsae strains identified by ssahaSNP (in comparison to AF16).

\begin{tabular}{|c|c|c|c|c|}
\hline & HK104 & VT847 & HK105 & PB800 \\
\hline $\begin{array}{l}\text { Sequence traces } \\
\text { examined }\end{array}$ & 13,632 & 14,976 & 2,112 & 384 \\
\hline $\begin{array}{l}\text { Traces aligned by } \\
\text { SSAHA }\end{array}$ & 7,530 & 9,213 & 1,680 & 123 \\
\hline $\begin{array}{l}\text { Total aligned base } \\
\text { pairs }\end{array}$ & $4,562,172$ & $5,761,972$ & $1,038,254$ & 75,508 \\
\hline $\begin{array}{l}\text { Total unique aligned } \\
\text { base pairs }\end{array}$ & $3,884,127$ & $4,327,725$ & 867,552 & 63,434 \\
\hline $\begin{array}{l}\text { Unique SNP loci } \\
\text { detected }\end{array}$ & 23,829 & 9,111 & 5,164 & 322 \\
\hline $\begin{array}{l}\text { Apparent SNP density } \\
\text { (per kb) }\end{array}$ & 6.13 & 2.11 & 5.95 & 5.08 \\
\hline
\end{tabular}

Total aligned base pairs include redundant matches due to sequence overlaps (between $15 \%$ and $25 \%$ ) in sequence data. The SNP density is based on the number of uniquely aligned base pairs. 
tion by far was $A(T)$ to $G(C)$, which accounted for $57.1 \%$ of all substitutions (Figure 1A).

\section{RFLP genotyping assays and validation of snip-SNPs}

We screened the SNPs predicted for HK104 for variants that altered the recognition site of a restriction enzyme, and thus might be amenable to restriction fragment length polymorphism (RFLP) genotyping. To make this a practical resource, we limited the analysis to 30 restriction enzymes from REBASE [23] that are reliable and inexpensive. Of 23,829 HK104 SNPs, some 4,700 $(19.72 \%)$ were predicted to alter the recognition site of at least one of the 30 restriction enzymes. To develop restriction fragment length polymorphism (RFLP) assays from these snip-SNPs, we designed PCR primers with a standard protocol and performed in silico digests of the

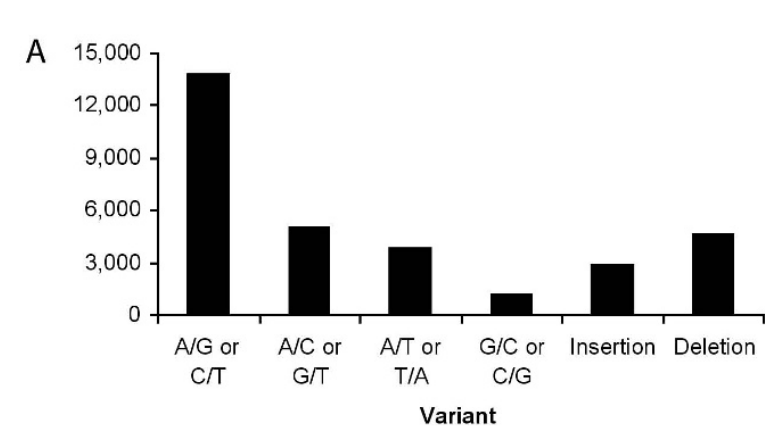

B

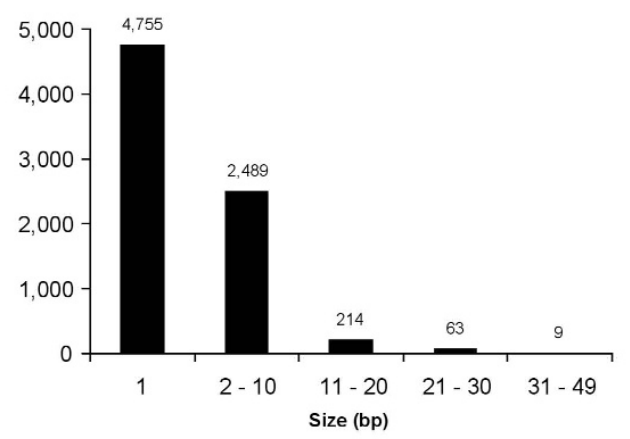

C

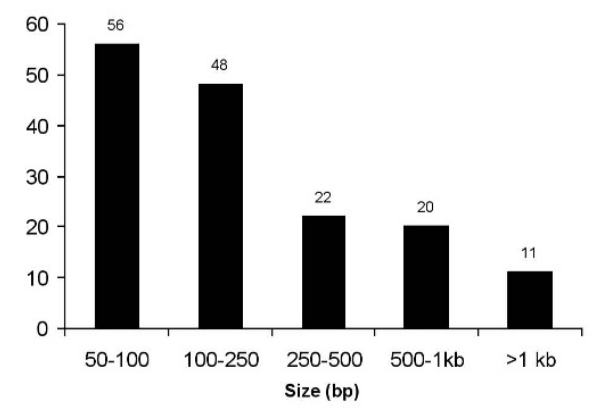

Figure 1 SNP discovery results for C. briggsae strain HK104. (A) Incidence of SNPs and small indels identified by ssahaSNP, by polymorphism type. (B) Distribution of small ( $<50$ bp) indels, by size. (C) Distribution of moderate (50-2,000 bp) indels, by size. resulting amplicons to infer the banding patterns for each strain. RFLPs not easily distinguishable on a gel, or SNPs on ultracontigs not yet included in the genetic map, were removed. Finally, we used assembly AGP information and BLAST alignment to obtain coordinates for each snipSNP on the cb3 sequence assembly. Our set contained 1,987 predicted RFLP assays from 1,362 snip-SNPs (some SNPs alter multiple RE sites) positioned on both the genetic and physical maps (see additional file 2). Another snip-SNP, bdP3, was identified in a separate study (see Methods and additional files 1 and 2).

We selected a total of 20 RFLP assays (between 3 and 4 for each chromosome) based on HindIII, DraI and SalI snip-SNPs for validation in AF16 and HK104 parental DNA (Table 2). Roughly a third of the assays (6) failed PCR in one or both strains in repeated attempts. Although we did not investigate the issue of PCR failure, it is possible that redesigning primers (by moving them out or in) and testing different PCR conditions may produce desired products in some cases. All of the 14 assays successfully gave rise to strain-specific RFLP banding patterns, validating the predicted snip-SNP (Table 2, Figure $2 \mathrm{~A}$ for two examples). Interestingly, two of these assays (cb55670 and cb20723) exhibited HK104 fragments that varied from in silico predictions, another possible consequence of unknown variants in this highly divergent strain. Consistent with $C$. briggsae clade structure [8], VT847 was not polymorphic (from AF16) for the snip-SNPs we examined.

\section{Indel Discovery}

The ssahaSNP program [24] was also able to detect insertion/deletion (indel) polymorphisms of 1-50 bp (Figure $1 \mathrm{~B})$. We used the parse_indel utility to extract 7,530 candidate indels (4,686 deletions and 2,844 insertions compared to AF16) for the HK104 strain. Most insertions and deletions detected by ssahaSNP were single base pair events; the largest was $49 \mathrm{bp}$. To identify larger insertion/ deletion events, we developed a customized algorithm called BreakPointRead that detects indels based on BLAST alignments between read sequences and the reference genome. First, the algorithm identifies "breakpoint reads" with alignment gaps of $10 \mathrm{bp}$ or larger compared to the reference sequence. Next, it analyzes the gap size and alignment orientations to infer the nature (insertion, deletion, inversion, etc.) and approximate size of sequence variation. When we applied BreakPointRead to the HK104 sequence traces, it identified 689 breakpoint reads suggestive of 635 underlying variants. We filtered the results to obtain insertion/deletion events between 50 and 2,000 bp. The resulting set contained 212 predicted indels (144 deletions and 68 insertions), the largest of which was a 1,707 bp deletion on chromosome IV (Figure $1 \mathrm{C}$ and additional file 3 ). 
Table 2: List of validated snip-SNPs by RFLP assays.

\begin{tabular}{|c|c|c|c|c|c|c|c|c|c|}
\hline \multirow[t]{2}{*}{ Chr } & \multirow[t]{2}{*}{ SNP id } & \multirow[t]{2}{*}{$\begin{array}{l}\text { Location } \\
\text { (cM) }\end{array}$} & \multirow[t]{2}{*}{ Ultra_contig } & \multirow[t]{2}{*}{ RE } & \multicolumn{2}{|c|}{$\begin{array}{l}\text { DNA fragments in AF16 } \\
\text { (bp) }\end{array}$} & \multicolumn{2}{|c|}{$\begin{array}{l}\text { DNA fragments in HK104 } \\
\text { (bp) }\end{array}$} & \multirow[t]{2}{*}{$\begin{array}{c}\text { Validation } \\
\text { type }\end{array}$} \\
\hline & & & & & Expected & Observed & Expected & Observed & \\
\hline \multirow[t]{5}{*}{1} & cb15251 & 13.45 & cb25.fpc4321 & Dral & 747 & 700 & 332 and 415 & 310 and 400 & * \\
\hline & cb55627 & 21.82 & cb25.fpc3441 & HindIII & 691 & 691 & 251 and 440 & 270 and 440 & * \\
\hline & cb55670 & 21.82 & cb25.fpc3441 & Dral & 312 and 437 & 749 & 749 & 312 and 437 & $* *$ \\
\hline & $\mathrm{cb} 650$ & 43.93 & cb25.fpc4140 & Sacl & 689 & 689 & 257 and 432 & 257 and 432 & * \\
\hline & (bhP27) & & & & & & & & \\
\hline \multirow[t]{3}{*}{2} & cb41028 & $11.88-13.1$ & cb25.fpc0011 & Dral & 332 and 419 & 332 and 400 & 751 & 800 & * \\
\hline & cb43091 & $21.28-26.58$ & cb25.fpc0058 & HindIII & 748 & 800 & 365 and 383 & 383 and 383 & * \\
\hline & cb64777 & 27.92-33.95 & cb25.fpc4206 & Dral & 325 and 424 & 325 and 400 & 749 & 749 & * \\
\hline \multirow[t]{3}{*}{3} & cb20723 & 12.46 & cb25.fpc4153 & Dral & $\begin{array}{r}20,196,220 \\
\text { and } 311\end{array}$ & $\begin{array}{l}230,311 \\
\text { and } 390\end{array}$ & $\begin{array}{r}20,196 \text { and } \\
531\end{array}$ & 230 and 800 & $* *$ \\
\hline & cb54953 & 21.77 & cb25.fpc2976 & HindIII & 751 & 800 & 368 and 383 & 380 and 400 & * \\
\hline & $\mathrm{cb} 40003$ & 31.14 & cb25.fpc0002 & Dral & 685 & PCR failure & 208 and 477 & 800 & \\
\hline \multirow[t]{3}{*}{4} & cb8971 & 7.88 & cb25.fpc4250 & Dral & 750 & PCR failure & 337 and 413 & 350 and 413 & \\
\hline & $\mathrm{cb} 48850$ & 20.02 & cb25.fpc1570 & HindIIII & 750 & 780 & 314 and 436 & 350 and 500 & * \\
\hline & cb56202 & $37.52-41.16$ & cb25.fpc3835 & Dral & $\begin{array}{r}60,190 \text { and } \\
489\end{array}$ & $\begin{array}{r}60,190 \text { and } \\
500\end{array}$ & 60 and 679 & 60 and 800 & * \\
\hline \multirow[t]{4}{*}{5} & cb39304 & $20.98-22.23$ & cb25.fpc4126 & HindIII & 742 & 790 & 286 and 456 & 310 and 470 & $*$ \\
\hline & cb39354 & $20.98-22.23$ & cb25.fpc4126 & Dral & 751 & PCR failure & 269 and 482 & PCR failure & \\
\hline & cb62 & $17.07-17.74$ & $\mathrm{cb} 25 . \mathrm{fpc} 4470$ & Dral & $\begin{array}{r}54,295 \text { and } \\
400\end{array}$ & PCR failure & 54 and 695 & 800 & \\
\hline & bdP3 & unknown & cb25.fpc0156 & Dral & 907 & 907 & 227 and 680 & 227 and 680 & * \\
\hline \multirow[t]{3}{*}{$x$} & cb20148 & 9.52 & cb25.fpc0045 & Dral & 660 & PCR failure & 171 and 489 & PCR failure & \\
\hline & cb40232 & $20.1-20.71$ & cb25.fpc0003 & HindIII & 237 and 473 & 300 and 473 & 710 & 850 & * \\
\hline & $\mathrm{cb} 6050$ & 24.07 & cb25.fpc4403 & Dral & 750 & PCR failure & 270 and 480 & 290 and 510 & \\
\hline
\end{tabular}

The snip-SNPs are arranged by chromosome and location. The corresponding ultracontigs are also listed. The expected and observed DNA fragments refer to products based on in silico analysis and actual experiments, respectively. The bdP3 location is unknown because the corresponding contig is unassembled. The validation type column marks snip-SNPs that were consistent with prediction $\left({ }^{*}\right)$ and those that differed significantly $\left.{ }^{* *}\right)$. RE: Restriction enzyme used to digest PCR amplified products.

\section{Validation of indels Medium indels}

We used the BreakPointRead algorithm to develop PCR fragment length polymorphism (PLP) assays for medium indels to facilitate high-throughput gene mapping. Of the 212 putative indels, we selected 40 for validation in AF16 and HK104 parental DNA (Figure 2B, C). Two other indels (bdP1 and bdP4) were also chosen for a similar analysis (see Methods and additional file 3). Ten of the 42 PLP assays failed PCR in one or both strains (Table 3). As mentioned earlier, some of these errors may be eliminated by redoing PCR using new primers. One assay (cbm16) resulted in PCR products that were quite large $(>1,500 \mathrm{bp})$ in both strains and therefore could not be accurately resolved on the gel. Of the remaining 31 assays that were successfully amplified, 29 confirmed the pres- 


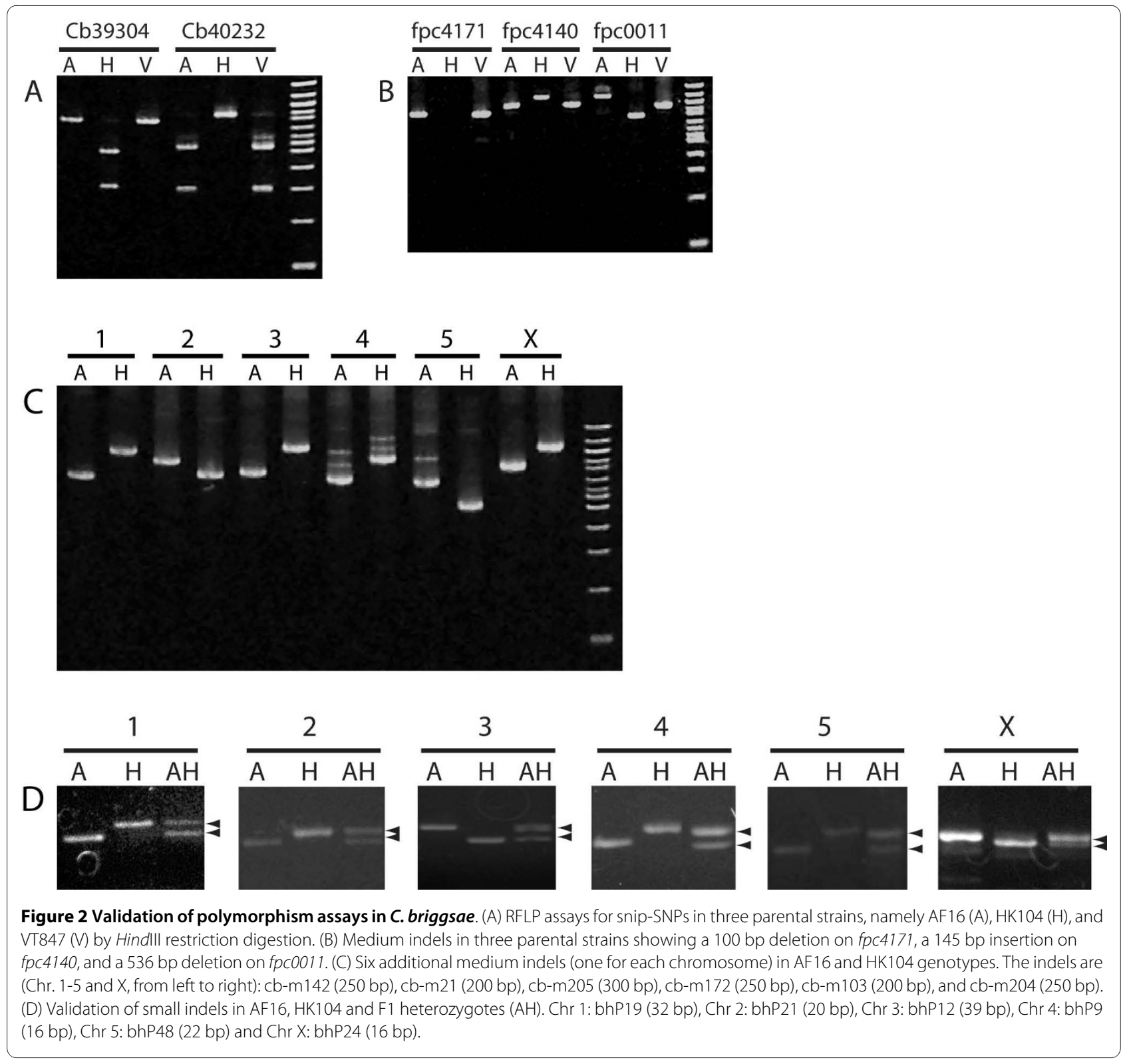

ence of polymorphism, 22 of which were similar in type and size as predicted (Table 3 ).

\section{Small indels}

We developed PLP assays for small ( $<50 \mathrm{bp}$ ) indels in AF16 and HK104 that were identified by ssahaSNP. To allow for gel resolution, we excluded indels smaller than 7 bp. This resulted in 436 assays that had 7-49 bp band size differences between AF16 and HK104 (see additional file 4). We tested 45 indels (between 4 and 9 for each chromosome) by PCR and found that except one (bhP44), for which HK104 amplification failed, all others could be successfully amplified (Table 4). A total of 32 indels showed bands of predicted sizes (Table 4, Figure 2D). Of the remaining 12, 1 showed no indel (i.e., identical PCR products in AF16 and HK104), 4 had multiple products (either due to PCR error, incorrect in silico predictions, or misassembly), and 7 showed PCR products that were inconsistent and unreliable (Table 4).

Altogether we experimentally confirmed 75 polymorphisms (14 snip-SNPs, 29 medium indels, and 32 small indels) on all six chromosomes (Figure 3, Table 5). The utility of these 'working' markers in genetic studies is demonstrated by successful mapping of several mutations that cause visible phenotypes. In two cases these mapping experiments also helped improve the genome sequence assembly. Specifically, the bhP42 contig fpc4184 was placed near the center of chromosome 1 and bhP18 contig $f p c 4010$ was reassigned to the left arm of chromosome 3 (see below). 
Table 3: List of medium indels tested by PCR.

\begin{tabular}{|c|c|c|c|c|c|c|c|c|c|c|}
\hline \multirow[t]{2}{*}{ Chr } & \multirow[t]{2}{*}{ Indel ID } & \multirow{2}{*}{$\begin{array}{l}\text { Location } \\
\text { (cM) }\end{array}$} & \multirow[t]{2}{*}{ Ultra_contig } & \multicolumn{2}{|c|}{ AF16 Amplicon (bp) } & \multicolumn{2}{|c|}{ HK104 Amplicon (bp) } & \multirow{2}{*}{$\begin{array}{l}\text { Predicted } \\
\text { indel }\end{array}$} & \multirow{2}{*}{$\begin{array}{c}\text { Validation } \\
\text { status }\end{array}$} & \multirow{2}{*}{$\begin{array}{c}\text { Validation } \\
\text { Type }\end{array}$} \\
\hline & & & & Predicted & Observed & Predicted & Observed & & & \\
\hline \multirow[t]{9}{*}{1} & cb-m142 & 12.6 & cb25.fpc4321 & 665 & 700 & 915 & 950 & $\begin{array}{c}250 \mathrm{bp} \\
\text { insertion }\end{array}$ & $\begin{array}{c}250 \mathrm{bp} \\
\text { insertion }\end{array}$ & * \\
\hline & $\mathrm{cb}-\mathrm{m} 2$ & 13.5 & cb25.fpc4321 & 1461 & Failed PCR & 1125 & Failed PCR & $\begin{array}{c}336 \mathrm{bp} \\
\text { deletion }\end{array}$ & Failed PCR & \\
\hline & $\mathrm{cb}-\mathrm{m} 5$ & 20.0 & cb25.fpc4171 & 774 & 750 & 674 & Failed PCR & $\begin{array}{c}100 \mathrm{bp} \\
\text { deletion }\end{array}$ & Failed PCR & \\
\hline & $\mathrm{cb}-\mathrm{m} 5$ & 20.0 & cb25.fpc4171 & 461 & 500 & 361 & 500 & $\begin{array}{l}100 \mathrm{bp} \\
\text { deletion }\end{array}$ & no indel & \\
\hline & cb-m6 & 43.9 & cb25.fpc4140 & 930 & 900 & 785 & 1000 & $\begin{array}{c}145 \mathrm{bp} \\
\text { deletion }\end{array}$ & $\begin{array}{c}100 \mathrm{bp} \\
\text { insertion }\end{array}$ & $* *$ \\
\hline & cb-m13 & unknown & cb25.fpc0078 & 504 & 420 & 385 & 400 & $\begin{array}{l}119 \mathrm{bp} \\
\text { deletion }\end{array}$ & $\begin{array}{c}20 \mathrm{bp} \\
\text { deletion }\end{array}$ & ** \\
\hline & cb-m14 & unknown & cb25.fpc4127 & 1000 & 1000 & 676 & 710 & $\begin{array}{l}324 \mathrm{bp} \\
\text { deletion }\end{array}$ & $\begin{array}{l}290 \mathrm{bp} \\
\text { deletion }\end{array}$ & * \\
\hline & cb-m146 & unknown & cb25.fpc4122 & 406 & 480 & 506 & 530 & $\begin{array}{c}100 \mathrm{bp} \\
\text { insertion }\end{array}$ & $\begin{array}{c}50 \mathrm{bp} \\
\text { insertion }\end{array}$ & $* *$ \\
\hline & $\mathrm{cb}-\mathrm{m} 12$ & unknown & cb25.fpc4127 & 921 & Failed PCR & 416 & 1000 & $\begin{array}{c}505 \mathrm{bp} \\
\text { deletion }\end{array}$ & Failed PCR & \\
\hline \multirow[t]{7}{*}{2} & cb-m21 & 24.2 & cb25.fpc0058 & 825 & 900 & 670 & 700 & $\begin{array}{l}155 \mathrm{bp} \\
\text { deletion }\end{array}$ & $\begin{array}{c}200 \mathrm{bp} \\
\text { deletion }\end{array}$ & * \\
\hline & cb-m16 & 37.4 & cb25.fpc4071 & 1097 & $>1500$ & 687 & $>1500$ & $\begin{array}{c}410 \mathrm{bp} \\
\text { deletion }\end{array}$ & unclear & \\
\hline & cb-m19 & 11.9-13.1 & cb25.fpc0011 & 1364 & 1013 & 828 & 750 & $\begin{array}{c}536 \mathrm{bp} \\
\text { deletion }\end{array}$ & $\begin{array}{c}263 \mathrm{bp} \\
\text { deletion }\end{array}$ & $* *$ \\
\hline & cb-m149 & $27.9-34.0$ & cb25.fpc4206 & 470 & 490 & 603 & 600 & $\begin{array}{c}133 \mathrm{bp} \\
\text { insertion }\end{array}$ & $\begin{array}{c}110 \mathrm{bp} \\
\text { insertion }\end{array}$ & * \\
\hline & cb-m26 & $27.9-34.0$ & cb25.fpc4206 & 678 & 700 & 407 & 410 & $\begin{array}{c}271 \mathrm{bp} \\
\text { deletion }\end{array}$ & $\begin{array}{c}290 \mathrm{bp} \\
\text { deletion }\end{array}$ & * \\
\hline & $\mathrm{cb}-\mathrm{m} 38$ & unknown & cb25.fpc4131 & 835 & 860 & 544 & 600 & $\begin{array}{c}291 \mathrm{bp} \\
\text { deletion }\end{array}$ & $\begin{array}{c}260 \mathrm{bp} \\
\text { deletion }\end{array}$ & * \\
\hline & bdP4 & unknown & cb25.fpc2441 & 1013 & $\sim 990$ & 903 & $\sim 880$ & $\begin{array}{c}110 \mathrm{bp} \\
\text { deletion }\end{array}$ & $\begin{array}{c}110 \mathrm{bp} \\
\text { deletion }\end{array}$ & * \\
\hline \multirow[t]{6}{*}{3} & cb-m46 & 6.1 & cb25.fpc2587 & 747 & 1020 & 574 & 750 & $\begin{array}{c}173 \mathrm{bp} \\
\text { deletion }\end{array}$ & $\begin{array}{l}270 \mathrm{bp} \\
\text { deletion }\end{array}$ & * \\
\hline & cb-m155 & 6.1 & cb25.fpc2587 & 1169 & Failed PCR & 1329 & Failed PCR & $\begin{array}{c}160 \mathrm{bp} \\
\text { insertion }\end{array}$ & Failed PCR & \\
\hline & $\mathrm{cb}-\mathrm{m} 48$ & 21.2 & cb25.fpc2976 & 1163 & 1200 & 644 & 1200 & $\begin{array}{l}519 \mathrm{bp} \\
\text { deletion }\end{array}$ & no indel & \\
\hline & cb-m205 & 25.8 & cb25.fpc4079 & 671 & 700 & 971 & 1000 & $\begin{array}{c}300 \mathrm{bp} \\
\text { insertion }\end{array}$ & $\begin{array}{c}300 \mathrm{bp} \\
\text { insertion }\end{array}$ & * \\
\hline & cb-m159 & 36.5 & cb25.fpc4224 & 463 & 500 & 655 & Failed PCR & $\begin{array}{c}192 \mathrm{bp} \\
\text { insertion }\end{array}$ & Failed PCR & \\
\hline & cb-m160 & $28.8-29.3$ & cb25.fpc2193 & 821 & 1000 & 1053 & 1200 & $\begin{array}{c}232 \mathrm{bp} \\
\text { insertion }\end{array}$ & $\begin{array}{c}200 \mathrm{bp} \\
\text { insertion }\end{array}$ & * \\
\hline
\end{tabular}


Table 3: List of medium indels tested by PCR. (Continued)

\begin{tabular}{|c|c|c|c|c|c|c|c|c|c|c|}
\hline & bdP1 & 31.3 & cb25.fpc0002 & 1723 & $\sim 1700$ & $\sim 1500$ & $\sim 1500$ & $\begin{array}{l}\sim 200 \mathrm{bp} \\
\text { deletion }\end{array}$ & $\begin{array}{c}200 \mathrm{bp} \\
\text { deletion }\end{array}$ & * \\
\hline \multirow[t]{9}{*}{4} & cb-m172 & 18.2 & cb25.fpc0039 & 650 & 700 & 904 & 950 & $\begin{array}{c}254 \mathrm{bp} \\
\text { insertion }\end{array}$ & $\begin{array}{c}250 \mathrm{bp} \\
\text { insertion }\end{array}$ & * \\
\hline & $\mathrm{cb}-\mathrm{m} 170$ & 20.6 & cb25.fpc4090 & 691 & 720 & 931 & Failed PCR & $\begin{array}{c}240 \mathrm{bp} \\
\text { insertion }\end{array}$ & Failed PCR & \\
\hline & $\mathrm{cb}-\mathrm{m} 171$ & 25.5 & cb25.fpc4132 & 381 & 450 & 476 & 550 & $\begin{array}{c}95 \mathrm{bp} \\
\text { insertion }\end{array}$ & $\begin{array}{c}100 \mathrm{bp} \\
\text { insertion }\end{array}$ & * \\
\hline & cb-m70 & 25.5 & cb25.fpc4132 & 594 & $>1600$ & 421 & 1600 & $\begin{array}{c}173 \mathrm{bp} \\
\text { deletion }\end{array}$ & $\begin{array}{c}\text { very large } \\
\text { indel }\end{array}$ & ** \\
\hline & cb-m176 & $37.5-41.2$ & cb25.fpc3835 & 732 & Failed PCR & 1014 & Failed PCR & $\begin{array}{c}282 \mathrm{bp} \\
\text { insertion }\end{array}$ & Failed PCR & \\
\hline & $\mathrm{cb}-\mathrm{m} 74$ & $37.5-41.2$ & cb25.fpc3835 & 677 & 1000 & 523 & 700 & $\begin{array}{l}154 \mathrm{bp} \\
\text { deletion }\end{array}$ & $\begin{array}{c}300 \mathrm{bp} \\
\text { deletion }\end{array}$ & ** \\
\hline & $\mathrm{cb}-\mathrm{m} 76$ & $42.4-43.0$ & cb25.fpc2328 & 709 & 900 & 527 & 550 & $\begin{array}{c}182 \mathrm{bp} \\
\text { deletion }\end{array}$ & $\begin{array}{l}350 \mathrm{bp} \\
\text { deletion }\end{array}$ & $* *$ \\
\hline & $\mathrm{cb}-\mathrm{m} 177$ & $9.2-9.8$ & cb25.fpc4118 & 801 & 800 & 946 & 900 & $\begin{array}{c}145 \mathrm{bp} \\
\text { insertion }\end{array}$ & $\begin{array}{c}100 \mathrm{bp} \\
\text { insertion }\end{array}$ & * \\
\hline & cb-m179 & unknown & cb25.fpc4331 & 383 & 450 & 500 & 520 & $\begin{array}{c}117 \mathrm{bp} \\
\text { insertion }\end{array}$ & $\begin{array}{c}70 \mathrm{bp} \\
\text { insertion }\end{array}$ & * \\
\hline \multirow[t]{4}{*}{5} & cb-m103 & 19.0 & cb25.fpc2220 & 600 & 700 & 454 & 500 & $\begin{array}{l}146 \mathrm{bp} \\
\text { deletion }\end{array}$ & $\begin{array}{c}200 \mathrm{bp} \\
\text { deletion }\end{array}$ & * \\
\hline & cb-m97 & 46.1 & cb25.fpc4109 & 591 & 705 & 392 & 490 & $\begin{array}{c}199 \mathrm{bp} \\
\text { deletion }\end{array}$ & $\begin{array}{c}215 \mathrm{bp} \\
\text { deletion }\end{array}$ & * \\
\hline & cb-m105 & $37.0-37.7$ & cb25.fpc4063 & 692 & 800 & 454 & 520 & $\begin{array}{l}238 \mathrm{bp} \\
\text { deletion }\end{array}$ & $\begin{array}{l}280 \mathrm{bp} \\
\text { deletion }\end{array}$ & * \\
\hline & cb-m104 & $37.0-37.7$ & cb25.fpc4063 & 942 & Failed PCR & 661 & Failed PCR & $\begin{array}{l}281 \mathrm{bp} \\
\text { deletion }\end{array}$ & Failed PCR & \\
\hline \multirow[t]{6}{*}{$x$} & cb-m197 & 3.5 & cb25.fpc4033 & 692 & 700 & 855 & 790 & $\begin{array}{c}163 \mathrm{bp} \\
\text { insertion }\end{array}$ & $\begin{array}{c}90 \mathrm{bp} \\
\text { insertion }\end{array}$ & * \\
\hline & cb-m204 & 17.4 & cb25.fpc4044 & 689 & 750 & 949 & 1000 & $\begin{array}{c}260 \mathrm{bp} \\
\text { insertion }\end{array}$ & $\begin{array}{c}250 \mathrm{bp} \\
\text { insertion }\end{array}$ & * \\
\hline & cb-m136 & 30.1 & cb25.fpc0829 & 1440 & 2000 & 645 & 750 & $\begin{array}{l}795 \mathrm{bp} \\
\text { deletion }\end{array}$ & $\begin{array}{l}1250 \mathrm{bp} \\
\text { deletion }\end{array}$ & * \\
\hline & cb-m127 & 34.1 & cb25.fpc2334 & 486 & 600 & 349 & 405 & $\begin{array}{l}137 \mathrm{bp} \\
\text { deletion }\end{array}$ & $\begin{array}{l}195 \mathrm{bp} \\
\text { deletion }\end{array}$ & * \\
\hline & cb-m126 & 34.1 & cb25.fpc2334 & 785 & Failed PCR & 648 & Failed PCR & $\begin{array}{c}137 \mathrm{bp} \\
\text { deletion }\end{array}$ & Failed PCR & \\
\hline & cb-m135 & $27.4-34.1$ & cb25.fpc0829 & 720 & 900 & 584 & Failed PCR & $\begin{array}{c}136 \mathrm{bp} \\
\text { deletion }\end{array}$ & Failed PCR & \\
\hline
\end{tabular}

The indels are arranged by chromosome and location. The corresponding ultracontigs are also listed. The "unknown" locations refer to unassembled contigs. The predicted indel sizes are based on in silico analysis of AF16 and HK104 amplicons. The validation type column marks indels that were consistent with prediction $\left(^{*}\right)$ and those that differed significantly $\left(^{* *}\right)$. 
Table 4: List of small indels tested by PCR.

\begin{tabular}{|c|c|c|c|c|c|c|c|}
\hline \multirow[t]{2}{*}{ Chr } & \multirow[t]{2}{*}{ Indel ID } & \multirow[t]{2}{*}{ Location (cM) } & \multirow[t]{2}{*}{ Ultra_contig } & \multirow{2}{*}{$\begin{array}{c}\text { Predicted } \\
\text { indel size (bp) }\end{array}$} & \multicolumn{2}{|c|}{ Predicted amplicon } & \multirow[t]{2}{*}{ Status } \\
\hline & & & & & AF16 & HK104 & \\
\hline \multirow[t]{7}{*}{1} & bhP41 & unknown & cb25.fpc4180 & 16 & 242 & 258 & $\begin{array}{l}\text { Inconsistent } \\
\text { products }\end{array}$ \\
\hline & bhP42 & unknown & cb25.fpc4184 & 15 & 252 & 267 & True \\
\hline & bhP19 & 0.7 & cb25.fpc2695 & 32 & 247 & 215 & True \\
\hline & bhP43 & 14 & cb25.fpc3857b & 12 & 245 & 257 & $\begin{array}{l}\text { Multiple } \\
\text { products }\end{array}$ \\
\hline & bhP7 & 28.6 & cb25.fpc2032 & 7 & 246 & 239 & True \\
\hline & bhP1 & 29.2 & cb25.fpc3441 & 10 & 250 & 260 & True \\
\hline & bhP29 & $\sim 52$ & cb25.fpc4140 & 17 & 248 & 231 & True \\
\hline \multirow[t]{9}{*}{2} & bhP20 & 2.4 & cb25.fpc4168 & 21 & 248 & 227 & $\begin{array}{l}\text { Inconsistent } \\
\text { products }\end{array}$ \\
\hline & bhP6 & $\sim 8$ & cb25.fpc0071 & 11 & 973 & 962 & True \\
\hline & bhP2 & $\sim 10$ & cb25.fpc3052a & 7 & 243 & 236 & True \\
\hline & bhP33 & $\sim 10$ & cb25.fpc3052a & 44 & 245 & 201 & $\begin{array}{l}\text { Inconsistent } \\
\text { products }\end{array}$ \\
\hline & bhP28 & $11.9-13.1$ & cb25.fpc0011 & 22 & 249 & 227 & $\begin{array}{l}\text { Inconsistent } \\
\text { products }\end{array}$ \\
\hline & bhP32 & $11.9-13.1$ & cb25.fpc0011 & 21 & 250 & 271 & True \\
\hline & bhP21 & $23.3-28.6$ & cb25.fpc0058 & 20 & 251 & 231 & True \\
\hline & bhP44 & $\sim 38$ & cb25.fpc1402a & 12 & 312 & 324 & $\begin{array}{l}\text { Failed PCR in } \\
\text { HK104 }\end{array}$ \\
\hline & bhP8 & 49.9 & cb25.fpc0305 & 18 & 249 & 231 & True \\
\hline \multirow[t]{8}{*}{3} & bhP18 & 0 & cb25.fpc4010 & 8 & 248 & 256 & True \\
\hline & bhP14 & 12.5 & cb25.fpc4153 & 22 & 241 & 219 & True \\
\hline & bhP38 & $16.8-17.5$ & cb25.fpc2187 & 44 & 249 & 293 & True \\
\hline & bhP12 & 21.2 & cb25.fpc2976 & 29 & 248 & 219 & True \\
\hline & bhP34 & 21.8 & cb25.fpc2976 & 7 & 246 & 239 & True \\
\hline & bhP39 & 25.7 & cb25.fpc0201 & 13 & 250 & 263 & $\begin{array}{l}\text { Multiple } \\
\text { products }\end{array}$ \\
\hline & bhP40 & 30 & cb25.fpc0002 & 20 & 250 & 270 & True \\
\hline & bhP10 & 35.4 & cb25.fpc4224 & 11 & 249 & 238 & No indel \\
\hline \multirow[t]{7}{*}{4} & bhP13 & $1.9-5.1$ & cb25.fpc3752 & 14 & 250 & 264 & True \\
\hline & bhP15 & 7.9 & cb25.fpc4250 & 20 & 250 & 270 & True \\
\hline & bhP45 & 17.6 & cb25.fpc4260 & 20 & 245 & 265 & True \\
\hline & bhP4 & $18.2-18.8$ & cb25.fpc0039 & 9 & 250 & 241 & $\begin{array}{l}\text { Inconsistent } \\
\text { products }\end{array}$ \\
\hline & bhP11 & 20.6 & cb25.fpc4090 & 18 & 251 & 233 & True \\
\hline & bhP9 & 31 & cb25.fpc1570 & 16 & 256 & 272 & True \\
\hline & bhP16 & 43.5 & cb25.fpc0107 & 21 & 251 & 230 & True \\
\hline
\end{tabular}


Table 4: List of small indels tested by PCR. (Continued)

\begin{tabular}{|c|c|c|c|c|c|c|c|}
\hline & bhP30 & 57.8 & cb25.fpc3052b & 20 & 248 & 262 & $\begin{array}{l}\text { Inconsisten } \\
\text { products }\end{array}$ \\
\hline & bhP46 & 57.8 & cb25.fpc3052b & 10 & 242 & 252 & $\begin{array}{l}\text { Multiple } \\
\text { products }\end{array}$ \\
\hline \multirow[t]{8}{*}{5} & bhP22 & 1.9 & cb25.fpc4095 & 14 & 249 & 263 & $\begin{array}{l}\text { Inconsisten } \\
\text { products }\end{array}$ \\
\hline & bhP31 & $2.5-3.2$ & cb25.fpc2114 & 24 & 244 & 268 & True \\
\hline & bhP47 & 9.6 & cb25.fpc2887a & 21 & 243 & 222 & True \\
\hline & bhP37 & 18.9 & cb25.fpc4470 & 10 & 251 & 261 & True \\
\hline & bhP5 & 26.7 & cb25.fpc0090 & 16 & 291 & 275 & True \\
\hline & bhP23 & 26.7 & cb25.fpc0090 & 22 & 250 & 228 & $\begin{array}{l}\text { Multiple } \\
\text { products }\end{array}$ \\
\hline & bhP48 & $40.2-40.8$ & cb25.fpc4063 & 22 & 249 & 271 & True \\
\hline & bhP24 & 56.9 & cb25.fpc0129 & 16 & 242 & 258 & True \\
\hline \multirow[t]{4}{*}{$x$} & bhP25 & 8.4 & cb25.fpc0045 & 32 & 250 & 218 & True \\
\hline & bhP36 & 13.6-16.3 & cb25.fpc4044b & 11 & 244 & 255 & True \\
\hline & bhP26 & $21-21.7$ & cb25.fpc0106 & 22 & 251 & 229 & True \\
\hline & bhP49 & 34.1 & cb25.fpc0829 & 14 & 250 & 264 & True \\
\hline
\end{tabular}

The table is organized similar to Table 3. The "Status" column shows whether an indel was correctly verified by PCR (True) or not (False). In all cases DNA sizes were determined by visual inspection.

\section{Linkage mapping studies using snip-SNPs and indels}

The snip-SNPs and indels were used to map a set of 12 mutations with easily recognizable phenotypes (e.g., Uncoordinated or Unc and Dumpy or Dpy) that were previously isolated in different laboratories (Table 6 and Methods). Except lin(bh25) and unc(sy5415), all other loci were uniquely assigned to linkage groups by standard 2factor mapping using known mutations that serve as reference (Table 6, also see [21]). The $d p y(s 1272)$, $u n c(s 1270)$, and $u n c(s a 997)$ are reference markers for LGIII, LGIV, and LGV, respectively. The remaining auto- somal loci are linked to C. elegans orthologs Cbr-lin-11 (LGI) and Cbr-unc-4 (LGII). Not only did the polymorphism-based mapping agree with phenotypic markerbased mapping (see $d p y(s y 5001)$ and $d p y$ (sy5148) in Figure $4 \mathrm{~A}, \mathrm{~B})$, it also helped to identify linkage groups of lin(bh25) (LGI, see Figure 4D and Table 6) and unc(sy5415) (LGV, see Table 6). In each of these cases a single cross with HK104 provided enough genomic DNA and usually one PCR per chromosome was sufficient to establish the linkage (using bulk-segregant approach, BSA). We also quantified DNA band intensities to deter-

Table 5: Summary of polymorphisms experimentally tested in this study.

\begin{tabular}{lllll}
\hline Category & Snip-SNP & Medium Indel & Small indel \\
\hline Attempted & 20 & 42 & 45 & 107 \\
\hline PCR failure cases & 6 & 10 & 1 & 17 \\
\hline $\begin{array}{c}\text { Successful PCR cases } \\
\text { Similar }\end{array}$ & 14 & 32 & 44 & $32(73 \%)$ \\
Different & $12(86 \%)$ & $22(69 \%)$ & 0 & 90 \\
False & 2 & 7 & 3 & 12 \\
\hline
\end{tabular}

A summary of data presented in Tables 2-4. The successful PCR cases are divided into three categories: similar (DNA fragments predicted correctly), different (DNA fragments that differed significantly from prediction), and false (no polymorphism). 


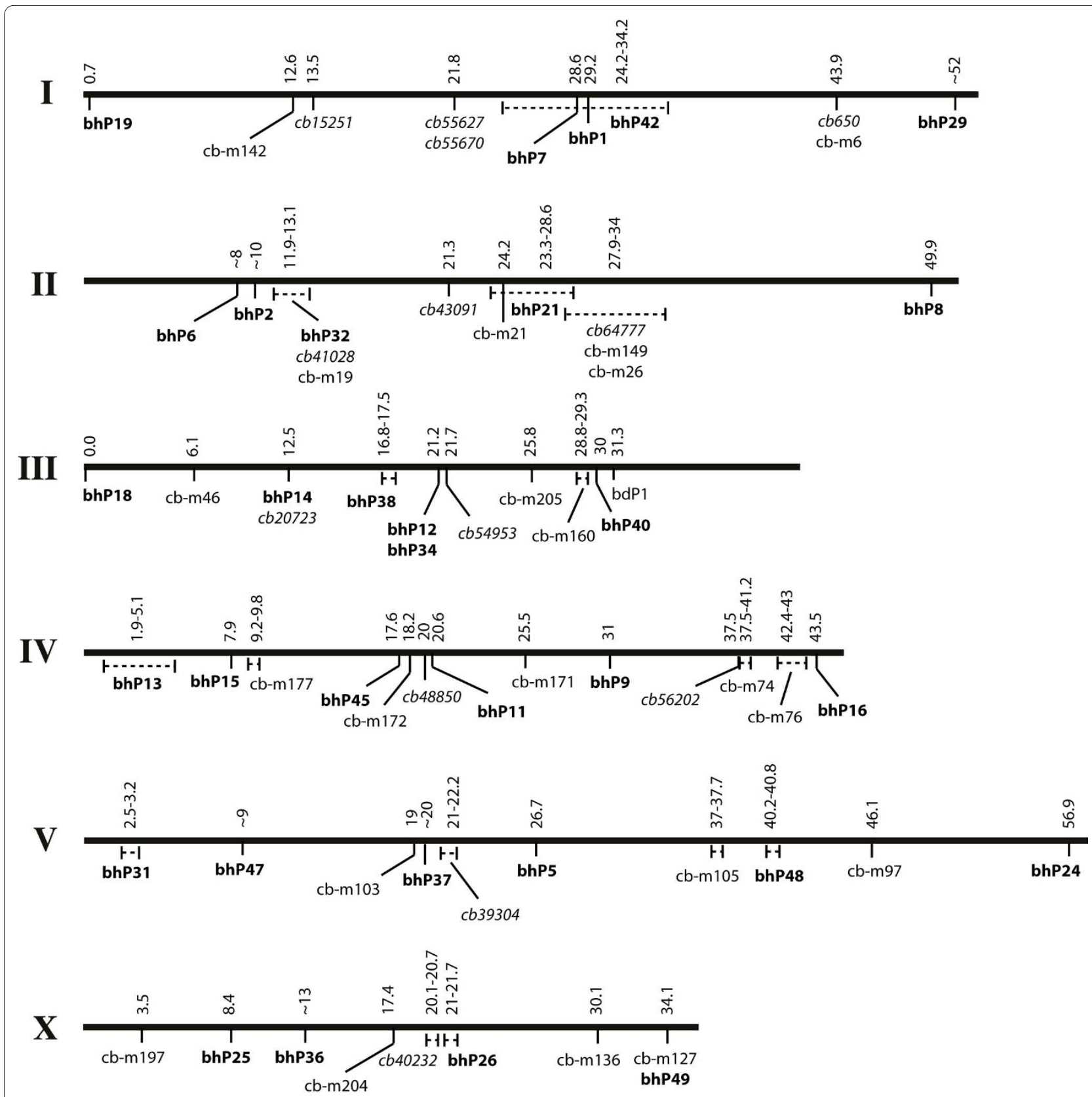

Figure 3 A polymorphism-based genetic linkage map of $\boldsymbol{C}$. briggsae. The map consists of 13 snip-SNPs (italics), 22 medium indels, and 32 small (bold) indels.

mine linkages as unitless linkage values (ULVs) (see Methods). As expected, for unlinked loci the ULV was one. In the case of $d p y$ (sy5148) the ULV for chromosome 2 indel (bhP21) was 2.7 suggesting a strong linkage (Figure 4C). Overall, these results demonstrate that polymorphism-based mapping can be used to quickly map new mutations in C. briggsae.

In addition to linking mutations to chromosomes we also investigated whether polymorphisms could be used in more precise mapping i.e., placing mutations in spe- cific chromosomal regions (left, right arms, or middle). We reasoned that by narrowing down genetic intervals of mutations it should be possible to identify potential candidates, including $\mathrm{C}$. elegans orthologs, thereby facilitating gene cloning by $\mathrm{RNAi}$ and transgene rescue approaches. To this end we used three medium indels on chromosome $\mathrm{X}$ to map dpy(sy5001). The ULVs for dpy(sy5001) suggest weak linkages to indels cb-m204 (left arm) and cb-m136 (right arm) and tight linkage to the middle indel cb-m197 (Figure 5A). Similar result was also 
Table 6: List of mutations used in polymorphism mapping experiments.

\begin{tabular}{|c|c|c|c|c|}
\hline Mutation & Phenotype & $\begin{array}{l}\text { Linkage group based } \\
\text { on phenotypic } \\
\text { markers }\end{array}$ & $\begin{array}{l}\text { Linked chromosome } \\
\text { and polymorphisms }\end{array}$ & Mutation source \\
\hline $\operatorname{lev}(s y 5440)$ & Lev-R, Unc & LGI (Cbr-lin-11) & 1 (bhP34) & Sternberg lab \\
\hline $\operatorname{lin}(b h 25)$ & Egl, Lin, Unc & $?$ & 1 (bhP1, bhP29, cb650) & Gupta lab \\
\hline$d p y(n m 4)$ & Dpy & LGII (Cbr-unc-4) & $2(\mathrm{bhP} 21)$ & Haag lab \\
\hline$d p y(\operatorname{sy} 5148)$ & Dpy & LGII (Cbr-unc-4) & 2 (bhP21) & Sternberg lab \\
\hline$d p y(s 1272)$ & Dpy & LGIII & $\begin{array}{l}3 \text { (bhP12, bhP14, } \\
\text { bhP18) }\end{array}$ & Baillie lab \\
\hline unc(sa972) & Unc, Sma & LGIII (dpy(s1272)) & 3 (bhP14, bhP18) & Thomas lab \\
\hline $\operatorname{lin}(b h 20)$ & Egl, Vul & LGIII (dpy(s1272)) & $\begin{array}{l}3 \text { (bhP14, bhP38, } \\
\text { bhP40) }\end{array}$ & Gupta lab \\
\hline unc(sy5422) & Unc & LGIV (unc(s1270)) & $\begin{array}{l}4 \text { (bhP9, bhP11, bhP15, } \\
\text { bhP16) }\end{array}$ & Sternberg lab \\
\hline unc(sa997) & Unc & LGV & $5(\mathrm{bhP} 24, \mathrm{bhP} 31)$ & Thomas lab \\
\hline unc(sy5415) & Unc & $?$ & 5 (bhP37) & Sternberg lab \\
\hline unc(sy5506) & Unc & LGX & $X(b h P 26)$ & Sternberg lab \\
\hline dpy(sy5001) & Dpy & LGX & $\begin{array}{l}X(b h P 36, c b-m 136, c b- \\
\text { m197, cb-m204) }\end{array}$ & Sternberg lab \\
\hline
\end{tabular}

Mutations are arranged by linkage group (LG) that corresponds to respective chromosome. For locations of polymorphism, refer to Figure 3. The mutant phenotypes are - Lev-R: Resistant to $1 \mathrm{mM}$ levamisole; Unc: Uncoordinated; Dpy: Dumpy; Egl: Egg-laying defective; Lin: Lineage defective; Sma: Small; Vul: Vulvaless. Question marks (?) refer to mutations that were not mapped by phenotypic markers.

obtained with the small indel bhP26 that is located close to cb-m197 and is strongly linked to dpy(sy5001) (data not shown).

Besides dpy(sy5001), we also mapped 4 autosomal mutations to sub-chromosomal regions using small indels. The results showed that $d p y(s 1272)$ is most strongly linked to bhP18, unc(sa972) to bhP14 and bhP18, and $\operatorname{lin}($ bh20) to bhP40 (all on chromosome 3) (Figure 5B). The unc(sy5422) appears to be located on the right arm of chromosome 4 (closer to the middle) since it shows strongest linkage to bhP9 (Figure $5 \mathrm{C}$ ).

The $C$. briggsae sequence assembly 'cb3' had placed the bhP18 contig $f p c 4010$ on the right arm of chromosome 3 $(\sim 36.5 \mathrm{mu})$. We found that this location was inconsistent with ULVs for $d p y(s 1272)$ and $u n c(s a 972)$ since both mutations are also linked to the left arm indel bhP14 (Figure $5 \mathrm{~B})$. This suggested that there could be a possible error in the sequence assembly. In a separate study Zhao et al. [25] used the SNP-based oligonucleotide array to map $d p y(s 1272)$ and found that the region corresponding to bhP18 is misassembled. Therefore, we have assigned bhP18, as well as $d p y(s 1272)$ and $u n c(s a 972)$, to the very end of the left arm of chromosome 3. Additional mapping using polymorphisms and visible markers will resolve the extent of misassembly.
In addition to the above BSA approach we also analyzed single F2 mutants segregating from a cross (termed single recombinant analysis or SRA) to determine recombination distances between polymorphisms and mutations. For this we used a multivulva mutation $\operatorname{lin}(s y 5353)$ and an Unc mutation unc(sy5506). The lin(sy5353) mutation is located on chromosome 1 since it is strongly linked to three small indels bhP1, bhP7 and bhP42 (B.P. Gupta, unpublished results). From a cross between lin(sy5353) and HK104 we picked 23 F2 mutant animals (46 chromosomes) and analyzed their DNA for the presence of bhP1. A single recombinant chromosome was recovered suggesting a recombination frequency of $\sim 2 \%$ between lin(sy5353) and bhP1. In the case of unc(sy5506) mutation, located on chromosome X, we analyzed $20 \mathrm{~F} 2$ mutant animals for the presence of indel bhP26. A total of 4 recombinant chromosomes were recovered (see additional file 5) suggesting that the two loci are $10 \%$ apart. These results demonstrate that SRA mapping protocol can be used in C. briggsae to localize mutations to subchromosomal regions and narrow down their genetic interval.

\section{Discussion and Conclusions}

We took a bioinformatics approach to identify polymorphisms in the C. briggsae genome and experimentally val- 


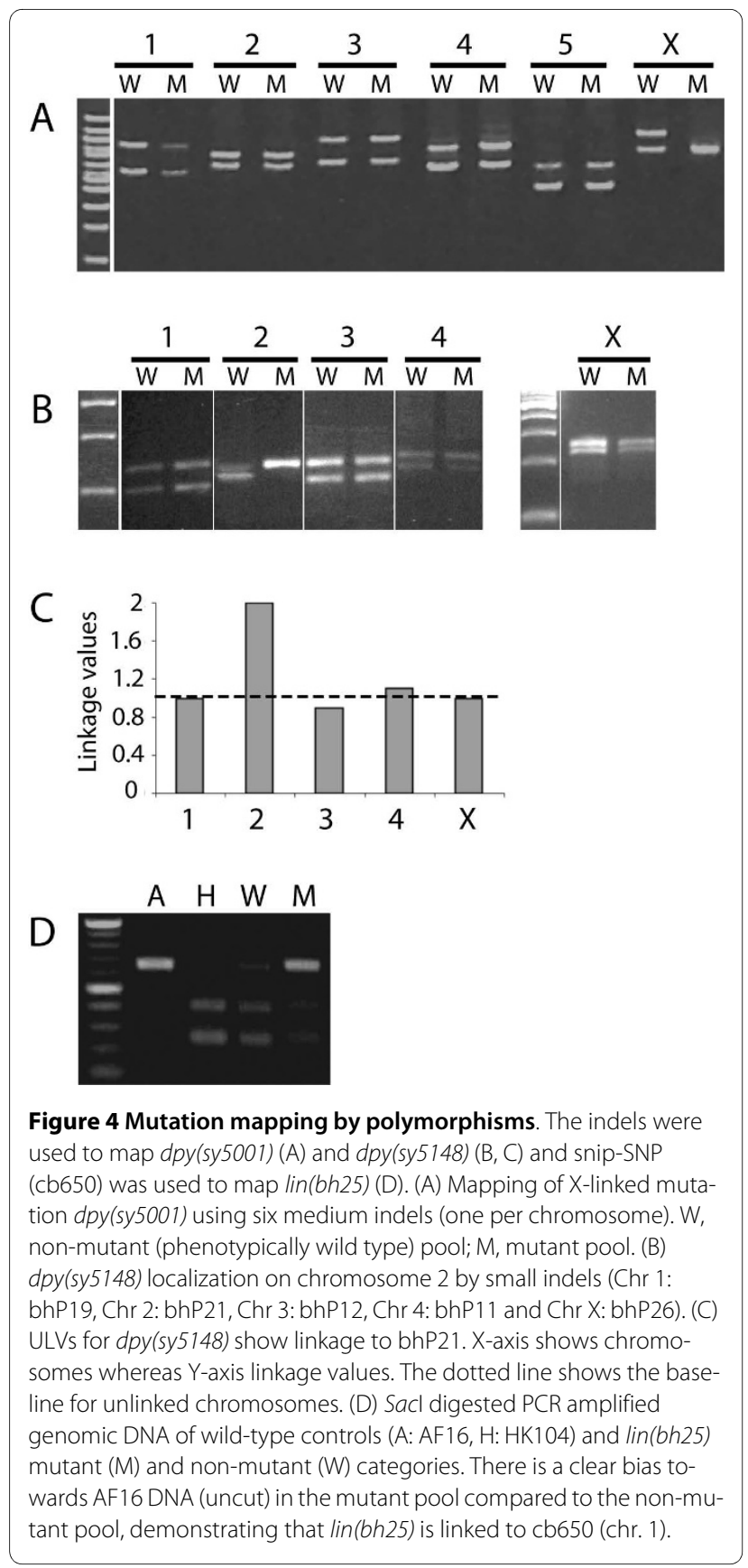

idated a small set of these to facilitate mapping of mutations. Comparison of AF16 (reference strain) to four other isolates (HK104, HK105, VT847 and PB800) revealed that HK104 is most polymorphic since it has the highest density of SNPs among all the strains. Altogether we identified $\sim 31,300$ polymorphisms $(23,800$ SNPs and 7,500 medium and small indels) between AF16 and HK104 that promise to be a valuable resource for mutation mapping and genome evolution studies. Roughly $20 \%$ of the SNPs are predicted to alter restriction enzyme sites (snip-SNPs) that could be detected by PCR followed by restriction digestion and agarose gel electrophoresis.
A total of 107 polymorphisms (20 snip-SNPs, 42 medium indels, and 45 small indels) that were experimentally tested, 66 (12 snip-SNPs, 22 medium indels, and 32 small indels) showed DNA fragments identical (or close) to in silico predictions (Table 5). Another 9 cases (2 snip-SNPs and 7 medium indels) were significantly different but nonetheless showed the presence of underlying variants. In 15 cases no polymorphism could be detected. Thus, excluding PCR failures (total 17), the success rate of correctly predicted polymorphisms was $73 \%$ (69-86\% range) (Table 5). This suggests that both ssahaSNP and BreakPointRead algorithms work equally efficiently regardless of the type of polymorphism in question. A similar study in C. elegans [26] showed that greater than 95\% of the polymorphisms predicted by the Polybayes program [27] are true. It remains to be seen whether the lower success rate in C. briggsae is due to intrinsic differences between the programs alone or if the quality of sequence data and assembly are additional contributing factors.

We used snip-SNPs and indels to map 12 mutations with visible phenotypes, and found that polymorphismbased mapping agreed with phenotypic marker-based results. Furthermore, it helped map two mutations, lin(bh25) and unc(sy5415), for which no prior genetic linkage data was available. Five mutations were also localized to sub-chromosomal regions. Thus our mapping resource can be used to rapidly map new mutations in $C$. briggsae. It is also relatively easy to validate additional polymorphisms if one needs a greater resolution. It should be pointed out that Hillier et al. [6] have validated another set of 9 snip-SNPs by sequencing during the process of $C$. briggsae genome sequence assembly (see additional file 6). Given the high density of such markers $(>2,000)$, it should be possible to map a mutation within a small genetic interval to facilitate molecular cloning (e.g., see [28]).

In addition to mapping mutations, SNPs and indels could also be used to improve the genetic linkage map of $C$. briggsae. The current C. briggsae sequence assembly, cb3, incorporates $90.2 \%(91.2 \mathrm{Mb})$ of the genome united into six chromosomes [6]. The remaining 9.8\% of sequences are tentatively associated with chromosomes. These unmapped regions could be integrated into chromosomes by polymorphism-based recombination mapping. We have successfully used this approach to place the contig fpc4184 in the vicinity of $f p c 3441$ (chromosome 1) based on the recombination distance of $5 \%$ between bhP42 (fpc4184) and bhP1 (fpc3441) (Figure 3) (A. Seetharaman, P. Cumbo, B. Nagagireesh and B. P. Gupta, manuscript submitted). In the other case, we have reassigned the bhP18 contig fpc4010 to the left arm of chromosome 3 based on its tight linkage to $d p y(s 1272)$ 


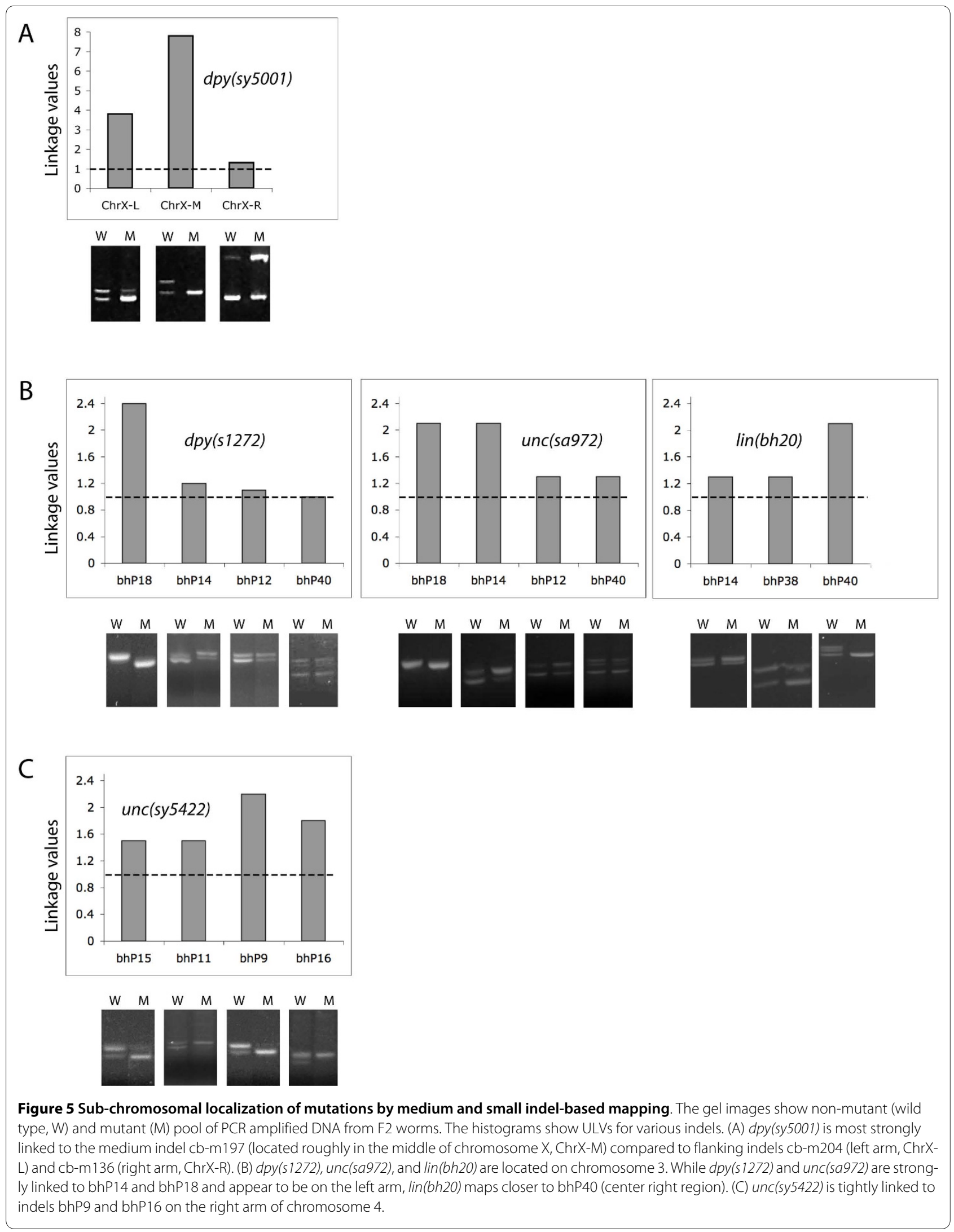


and $u n c(s a 972)$. Additional snip-SNPs and indels could further refine the locations of these contigs.

\section{Methods}

\section{Strains and culture conditions}

All strains were maintained at $22^{\circ} \mathrm{C}$. The general methods of culturing nematodes are previously described [29]. AF16 is the wild type genetic background for all strains. The four other wild-type C. briggsae isolates that were used for polymorphism discovery are HK104, HK105, VT847, and PB800. The HK104 strain was used in all mapping experiments. Various mutations used in this study are: $\quad c s p-1(s a 972), \quad d p y(n m 4), \quad d p y(s 1272)$, dpy(sy5001), dpy(sy5148), $\quad \operatorname{lev}(s y 5440), \quad \operatorname{lin}(b h 20)$, lin(bh25), lin(sy5353), unc(sa997), unc(sy5415), $u n c(s y 5422)$, and unc(sy5506).

\section{In silico predictions of polymorphisms \\ SNPS}

SNP discovery was performed on 13,632 shotgun sequence traces from strains HK104, VT847, HK105, and PB800. The ssahaSNP program (version SSAHA2) [24] was used to call SNPs due to its robust and efficient performance; only polymorphisms with quality scores above the minimum threshold were accepted. We also tested Polyphred (v5.04) [30] and PolyBayes (v3.0) [27] programs but found that only ssahaSNP could efficiently handle the entire read set and reference genome sequences as input. For a reference sequence the cb25 genome sequence assembly, which is based on strain AF16 and organized into ultra (fingerprint) contigs, was obtained from Wormbase. Flanking sequences for predicted SNPs were repeat-masked to lower case using the RepeatMasker program (v3.1.5) [31] with a customized $C$. briggsae repeat library.

The HK104 SNPs were positioned on the cb25 sequence assembly during SNP discovery. To position them on the newer cb3 sequence assembly, which is by chromosome, we obtained the assembly AGP files from Wormbase. SNP positions were inferred based on the coordinates and orientation of their cb25 ultracontig. SNPs on cb3-unmapped ultracontigs were mapped by WU-BLAST v2.0 (Gish, W., personal communication) alignment of their flanking sequences. Some 699 SNPs could not be positioned on the cb3 assembly by either method.

\section{Indels}

Candidate AF16-HK104 indels were extracted from HK104 sequence traces using the parse_indel utility of ssahaSNP. In the HK104 set, the largest indel event identified by ssahaSNP was $49 \mathrm{bp}$. To identify larger insertion/ deletion variants we implemented BreakPointRead, a custom algorithm that detects structural variations (insertions, deletions, inversions, and copy number variants) spanned by individual sequence traces. Traces were aligned to the reference genome (cb25) using WUBLAST v2.0 (Gish, W., personal communication), and screened for alignments with "gaps" of $>=10 \mathrm{bp}$. The alignment patterns of such "breakpoint reads" were used to infer the type and size of polymorphism. Predicted insertions and deletions were set aside for assay development.

\section{bdP polymorphisms}

The $b d P$ polymorphisms described in this study (snipSNP $b d P 3$ and medium indels $b d P 1$ and $b d P 4$ ) were identified in the laboratory of SEB. The snip-SNP $b d P 3$ was earlier used in a study involving ray pattern variation in C. briggsae [32].

\section{Development of RFLP and PCR Length Polymorphism (PLP) assays}

SNPs were screened for substitutions that altered the recognition sequence of restriction enzymes using the Bio:::Restriction::Analysis library of BioPerl [33]. The analysis was limited to 30 restriction enzymes from REBASE [23] known to be reliable and inexpensive. PCR assays were designed (amplicon sizes of 500 to $1000 \mathrm{bp}$, primer Tm's of $54-56^{\circ} \mathrm{C}$ ) using a local installation of the primer 3 program [34]. In silico fragment analysis of the PCR products was performed to predict band sizes for AF16 and HK104; assays with more than 4 bands in either strain were removed.

In the case of small indels (7-49 bp), primers were selected to generate AF16 amplicon sizes within the range of 200 and $400 \mathrm{bp}$. For medium indels $(50-2,000$ $\mathrm{bp})$, primers flanking each indel and specifying an AF16 amplicon size of 300-800 bp were selected.

\section{PCR}

In all experiments the genomic DNA from F2 worms (derived from a cross between AF16 and HK104 animals) was used as a PCR template. In some control experiments genomic DNA from F1 heterozygous animals was also used. PCR results that gave rise to unexpected or no products were repeated at least twice. In some cases we also tested different annealing temperatures. Those that consistently failed were termed as "PCR failure".

For RFLP and medium indel assays, $10 \mathrm{ng}$ dry PCR primers (IDT, Coralville, IA) were resuspended into 40 $\mu \mathrm{M}$ in a 96-well format. Our PCR mixture consisted of $300 \mathrm{pg}$ genomic DNA, $0.1 \mu \mathrm{M}$ PCR of each up and down primer, 0.02 U Platinum Taq (Invitrogen), $83.3 \mu \mathrm{M}$ per base dNTPs, $2.92 \mu \mathrm{M} \mathrm{MgCl}_{2}, 10 \times$ Buffer (16.7 mM Tris$\mathrm{HCl} \mathrm{pH} \mathrm{8.4,} 41.67 \mathrm{mM} \mathrm{KCl}$ - Invitrogen PCR Kit), and 4.2\% DMSO. Amplification was carried out in a PerkinElmer 384 PCR plate containing $12 \mu \mathrm{l}$ of $1 \times$ PCR mixture. The 384-well plate was sealed with Microseal A (M) Research) before carrying out the PCR. After initial heat 
denaturation step $\left(95^{\circ} \mathrm{C}-2 \mathrm{~min}\right.$.) we used a fixed 35 cycles PCR $\left(94^{\circ} \mathrm{C}-10 \mathrm{sec} ., 58^{\circ} \mathrm{C}-20 \mathrm{sec} ., 68^{\circ} \mathrm{C}-30 \mathrm{sec}\right.$. and a final extension $\left(68^{\circ} \mathrm{C}\right.$ for $10 \mathrm{~min}$.). PCR products were analyzed on $10 \%$ polyacrylamide gels that consisted of $33.3 \%$ of $29: 1$ acrylamide:bis (Biorad), $10 \%$ of $10 \times \mathrm{TBE}$, $15 \%$ of glycerol, $40.2 \% \mathrm{H}_{2} 0,3.4 \times 10^{-2} \%$ TEMED (Int'l Biotech), and $1.42 \%$ of $10 \%$ APS. Gels were stained with SYBR green (Invitrogen) and inspected over a UV light box at $254 \mathrm{~nm}$.

To detect small indels ( $<50 \mathrm{bp}$ ) we used a standard 35 cycles PCR $\left(94^{\circ} \mathrm{C}-10\right.$ sec., $48^{\circ} \mathrm{C}-30$ sec., $72^{\circ} \mathrm{C}-60$ sec. $)$ for all amplifications. For BSA-based mapping the genomic DNA was prepared from 25 adults in a $10 \mu \mathrm{l}$ lysis buffer (consisting of $50 \mathrm{mM} \mathrm{KCl}, 10 \mathrm{mM}$ Tris $\mathrm{pH} 8.2$, $2.5 \mathrm{mM} \mathrm{MgCl}, 0.45 \%$ Tween 20, 0.45\% NP40, $0.001 \%$ Gelatin, and $30 \mu \mathrm{g}$ Proteinase K). The mixture was frozen at $80^{\circ} \mathrm{C}$ (at least $30 \mathrm{~min}$ ) and then placed in a thermal cycler for $1 \mathrm{hr}$ incubation at $60^{\circ} \mathrm{C}$ followed by $15 \mathrm{~min}$ heat inactivation at $95^{\circ} \mathrm{C}$. The resulting genomic DNA was diluted to $25 \mu \mathrm{l}$ using sterile distilled water and stored at $-20^{\circ} \mathrm{C}$. The PCR mixture $(25 \mu \mathrm{l})$ contained $1 \mu \mathrm{l}$ genomic DNA, 1 $\mu \mathrm{l}$ each of up and down primers, $1 \mu \mathrm{l}$ dNTPs, $2.5 \mu \mathrm{l}$ NEB ThermoPol 10× PCR buffer, $0.2 \mu$ l NEB Taq Enzyme, and $18.3 \mu \mathrm{l}$ sterile distilled PCR grade water. For SRA-based mapping, single worms were placed in $5 \mu$ lysis buffer and processed as above. The lysed samples were used as DNA templates in PCR experiments. The amplified products were first analyzed on 1\% agarose gel (Invitrogen UltraPure, Catalog \#15510-027). Successful amplifications were subsequently examined on a $4 \%$ high-resolution agarose gel (Invitrogen UltaPure Agarose-1000, Catalog \#10975-035) to determine the presence of indels.

\section{Mutation mapping}

We picked 12 strains for linkage mapping studies (Table 6). The mutations were obtained from EMS (ethyl methane sulfonate) mutagenesis screens in an AF16 genetic background in various laboratories. The strains were outcrossed several times (3 or more). For mapping, mutant hermaphrodites were crossed with HK104 males and the genomic DNA from 20 F2 animals (wild type and mutant separately) was prepared as described in the previous section. The linkage was determined by PCR using protocols established for control experiments.

\section{Linkage and ULV analysis}

To determine the linkage of a mutation to a chromosome, we initially relied on the visual inspection of DNA band intensities on Ethidium bromide-stained agarose gels. Subsequently, in indel-based mapping experiments, we calculated linkages as unitless values (ULVs) for an unbiased analysis. The mean intensities of DNA bands were measured by NIH ImageJ software (version 1.41o; [35]) using Measure tool under Analyze menu. For each muta- tion a ratio of band intensities in the "mutant" lane was calculated by dividing the mean intensities of the AF16 bands by the mean intensities of the HK104 bands. This ratio was termed as the ULV. As expected, ULVs were one for unlinked mutations and higher for linked mutations.

\section{Genetic positions of polymorphisms}

The genetic positions of snip-SNPs and indels in this study correspond to nearest SNPs that were experimentally validated (D.C.K. and R.D.M., unpublished). These 'verified' SNPs (400 in total) were genotyped in RILs derived from two independent crosses between AF16, HK104 and VT847 (AF16 $\times$ HK104 and AF16 $\times$ VT847). The details are available on the C. briggsae SNP Research Facility website [36].

\section{Polymorphisms and sequence data availability}

All identified snip-SNPs, indels and PCR primers, confirmed or otherwise, are accessible via the C. briggsae resource website (see "Polymorphism" link) [21]. The website also contains sequence reads of all four species (HK104, VT847, HK105, and PB800). The sequence directories are organized into Polyphred structure and contain additional files (such as read quality). Additional information on polymorphism discovery using sequence data can be obtained from the Washington University $C$. briggsae SNP Research Facility website [36]. The confirmed polymorphisms have also been submitted to Wormbase [37].

\section{List of abbreviations}

BSA: Bulk segregant analysis; Indel: Insertion-deletion; PLP: PCR fragment length polymorphism; RIL: Recombinant inbred line; RFLP: Restriction fragment length polymorphism; SNP: Single nucleotide polymorphism; SRA: Single recombinant analysis; ULV: Unitless linkage value.

\section{Additional material}

Additional file 1 List of SNPs in C. briggsae. An Excel file containing 23,829 computationally identified SNPs in AF16 and HK104

Additional file $\mathbf{2}$ List of snip-SNPs and RFLP assays. An Excel file containing 1988 SNPs that alter restriction enzyme site (snip-SNPs) along with PCR primers and predicted digestion patterns in AF16 and HK104.

Additional file $\mathbf{3}$ List of medium indels and PLP assays. An Excel file containing 214 medium indels along with PCR primers and predicted DNA fragments in AF16 and HK104.

Additional file $\mathbf{4}$ List of small indels and PLP assays. The Excel file containing 436 small indels along with PCR primers and predicted DNA fragments in AF16 and HK104.

Additional file 5 Mapping of unc(sy5506) mutation by single recombinant analysis (SRA). Twenty single F2 mutant animals were individually examined by PCR for the presence of indel bhP26. Four of these (\#7, \#10, $\# 16$, and \#17) were found to be recombinants, as judged by the presence of two bands on the agarose gel (corresponding to AF16 and HK104 DNA).

Additional file 6 C. briggsae snip-SNPs identified prior to this work. An Excel file containing nine C. briggsae snip-SNPs that were validated by Hillier et al. [6]. 


\section{Authors' contributions}

The C. briggsae Advisory Group (BPG, ESH, HMC, RDM, and SEB) planned the project and collected the genetic and financial resources to perform the work. DCK and RDM carried out bioinformatics searches. DCK designed snip-SNP and PLP assays. JES, BT and KH performed PCR experiments to validate polymorphisms and map mutations. BT and BPG calculated ULVs for mutations linked to medium and small indels. bdP polymorphisms were identified and validated in the laboratory of SEB. BPG wrote the paper with input from ESH, HMC, SEB, DCK, and JES. All authors read and approved the final manuscript.

\section{Acknowledgements}

The authors dedicate this work to the memory of our dear colleague and friend, Dr. Raymond D. Miller. We thank Prateek Goyal and Nagagireesh Bojanala for assistance in mapping some of the mutants. We also thank Paul Sternberg (California Institute of Technology), David Baillie (Simon Fraser), and James Thomas (University of Washington Seattle) for sharing strains and unpublished data on mutant isolation and genetic mapping. Many of the oligos were obtained from the MOBIX lab at McMaster University. This work was supported by a grant from National Institutes of Health (R24 GM075101) to RDM and BPG.

\section{Author Details}

'Department of Genetics, Washington University School of Medicine, St. Louis, Missouri 63110, USA, ${ }^{2}$ Department of Biology, McMaster University, Hamilton, ON L8S 4K1, Canada, ${ }^{3}$ Department of Biological Sciences, Wright State University, Dayton, OH 45435, USA, ${ }^{4}$ Department of Molecular Genetics, Ohio State University, Columbus, OH 43210, USA and ${ }^{5}$ Department of Biology, University of Maryland, College Park, MD 20742, USA

Received: 19 January 2010 Accepted: 13 April 2010

Published: 13 April 2010

\section{References}

1. Gupta BP, Johnsen R, Chen N: Genomics and biology of the nematode Caenorhabditis briggsae. In WormBook Community TCeR: WormBook; 2007.

2. Stein LD, Bao Z, Blasiar D, Blumenthal T, Brent MR, Chen N, Chinwalla A, Clarke L, Clee C, Coghlan A, et al:: The genome sequence of Caenorhabditis briggsae: a platform for comparative genomics. PLOS Biol 2003, 1(2): E45.

3. Antoshechkin I, Sternberg PW: The versatile worm: genetic and genomic resources for Caenorhabditis elegans research. Nat Rev Genet 2007, 8(7):518-532.

4. Cutter AD: Divergence times in Caenorhabditis and Drosophila inferred from direct estimates of the neutral mutation rate. Mol Biol Evol 2008, 25(4):778-786

5. The InParanoid database [http://inparanoid.sbc.su.se/cgi-bin/index.cgi]

6. Hillier LW, Miller RD, Baird SE, Chinwalla A, Fulton LA, Koboldt DC, Waterston RH: Comparison of C. elegans and C. briggsae Genome Sequences Reveals Extensive Conservation of Chromosome Organization and Synteny. PLOS Biol 2007, 5(7):e167.

7. Graustein A, Gaspar JM, Walters JR, Palopoli MF: Levels of DNA polymorphism vary with mating system in the nematode genus caenorhabditis. Genetics 2002, 161(1):99-107.

8. Cutter AD, Felix MA, Barriere A, Charlesworth D: Patterns of nucleotide polymorphism distinguish temperate and tropical wild isolates of Caenorhabditis briggsae. Genetics 2006, 173(4):2021-2031

9. Chen PJ, Cho S, Jin SW, Ellis RE: Specification of germ cell fates by FOG-3 has been conserved during nematode evolution. Genetics 2001 158(4):1513-1525

10. de Bono M, Hodgkin J: Evolution of sex determination in Caenorhabditis: unusually high divergence of tra-1 and its functional consequences. Genetics 1996, 144:587-595.

11. Hansen D, Pilgrim D: Molecular evolution of a sex determination protein. FEM-2 (pp2c) in Caenorhabditis. Genetics 1998, 149:1353-1362.

12. Wang $X$, Chamberlin HM: Evolutionary innovation of the excretory system in Caenorhabditis elegans. Nat Genet 2004, 36(3):231-232.

13. Streit A, Li W, Robertson B, Schein J, Kamal IH, Marra M, Wood WB: Homologs of the Caenorhabditis elegans masculinizing gene her-1 in C. briggsae and the filarial parasite Brugia malayi. Genetics 1999, 152(4):1573-1584
14. Haag ES, Wang S, Kimble J: Rapid coevolution of the nematode sexdetermining genes fem-3 and tra-2. Curr Biol 2002, 12(23):2035-2041.

15. Rudel D, Kimble J: Evolution of discrete Notch-like receptors from a distant gene duplication in Caenorhabditis. Evol Dev 2002, 4(5):319-333.

16. Stothard $P$, Hansen D, Pilgrim D: Evolution of the PP2C family in Caenorhabditis: rapid divergence of the sex-determining protein FEM2. J Mol Evol 2002, 54:267-282

17. Kuwabara PE: Interspecies comparison reveals evolution of contro regions in the nematode sex-determining gene tra-2. Genetics 1996, 144(2):597-607.

18. Inoue T, Ailion M, Poon S, Kim HK, Thomas JH, Sternberg PW: Genetic analysis of dauer formation in Caenorhabditis briggsae. Genetics 2007, 177(2):809-818

19. Kelleher JF, Mandell MA, Moulder G, Hill KL, L'Hernault SW, Barstead R, Titus MA: Myosin VI is required for asymmetric segregation of cellular components during C. elegans spermatogenesis. Curr Biol 2000, 10(23):1489-1496

20. Hill RC, de Carvalho CE, Salogiannis J, Schlager B, Pilgrim D, Haag ES: Genetic flexibility in the convergent evolution of hermaphroditism in Caenorhabditis nematodes. Dev Cell 2006, 10(4):531-538.

21. Caenorhabditis briggsae Research Resource [http://www.briggsae.org]

22. Wormbase FTP site [ftp///ftp.wormbase.org/pub/wormbase/genomes/

23. Roberts RJ, Vincze T, Posfai J, Macelis D: REBASE--enzymes and genes for DNA restriction and modification. Nucleic Acids Res 2007:D269-270

24. Ning Z, Cox AJ, Mullikin JC: SSAHA: a fast search method for large DNA databases. Genome Res 2001, 11(10):1725-1729.

25. Zhao Z, Flibotte S, Murray Jl, Blick D, Boyle TJ, Gupta B, Moerman DG Waterston RH: New tools for investigating the comparative biology of Caenorhabditis briggsae and C. elegans. Genetics 2010, 184(3):853-863.

26. Wicks SR, Yeh RT, Gish WR, Waterston RH, Plasterk RH: Rapid gene mapping in Caenorhabditis elegans using a high density polymorphism map. Nat Genet 2001, 28(2):160-164

27. Marth GT, Korf I, Yandell MD, Yeh RT, Gu Z, Zakeri H, Stitziel NO, Hillier L, Kwok PY, Gish WR: A general approach to single-nucleotide polymorphism discovery. Nat Genet 1999, 23(4):452-456.

28. Guo Y, Lang S, Ellis RE: Independent recruitment of $F$ box genes to regulate hermaphrodite development during nematode evolution. Curr Biol 2009, 19(21):1853-1860

29. Brenner S: The genetics of Caenorhabditis elegans. Genetics 1974, 77:71-94.

30. Nickerson DA, Tobe VO, Taylor SL: PolyPhred: automating the detection and genotyping of single nucleotide substitutions using fluorescencebased resequencing. Nucleic Acids Res 1997, 25(14):2745-2751.

31. RepeatMasker [http://www.repeatmasker.org]

32. Baird SE, Davidson CR, Bohrer JC: The genetics of ray pattern variation in Caenorhabditis briggsae. BMC Evol Biol 2005, 5(1):3.

33. Stajich JE, Block D, Boulez K, Brenner SE, Chervitz SA, Dagdigian C, Fuellen G, Gilbert JG, Korf I, Lapp H, et al.: The Bioperl toolkit: Perl modules for the life sciences. Genome Res 2002, 12(10):1611-1618.

34. Rozen S, Skaletsky H: Primer3 on the WWW for general users and for biologist programmers. Methods Mol Biol 2000, 132:365-386.

35. ImageJ: Image processing and analysis program [http:// rsb.info.nih.gov/nih-image

36. C. briggsae SNP Research Facility [http://snp.wustl.edu/snp-research/cbriggsae/genetic-map-of-c-briggsae.html

37. Wormbase: The biology and genome of C. elegans [http:// www.wormbase.org]

doi: 10.1186/1471-2164-11-236

Cite this article as: Koboldt et al., A toolkit for rapid gene mapping in the nematode Caenorhabditis briggsae BMC Genomics 2010, 11:236 